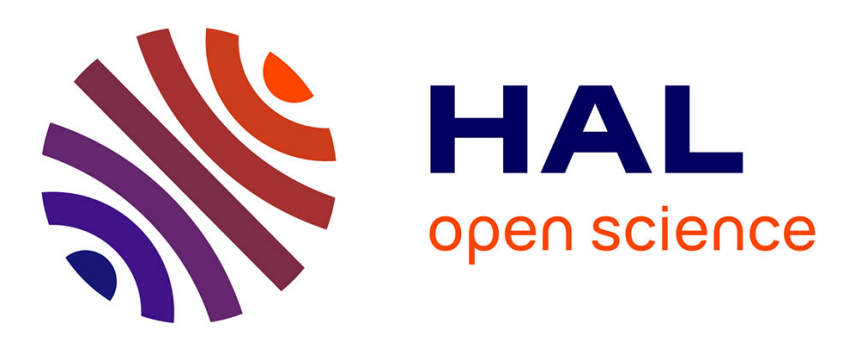

\title{
L'avenir des négociations collectives
}

Franck Biétry, Marc Morin, Jacques Freyssinet, Jacques Rojot, Christian Thuderoz

\section{To cite this version:}

Franck Biétry, Marc Morin, Jacques Freyssinet, Jacques Rojot, Christian Thuderoz. L'avenir des négociations collectives. RIMHE: Revue Interdisciplinaire Management, Homme(s) \& Entreprise, 2015, 16 (2), pp.3-17. 10.3917/rimhe.016.0003 . hal-02007486

\section{HAL Id: hal-02007486}

\section{https://hal-normandie-univ.archives-ouvertes.fr/hal-02007486}

Submitted on 11 Apr 2019

HAL is a multi-disciplinary open access archive for the deposit and dissemination of scientific research documents, whether they are published or not. The documents may come from teaching and research institutions in France or abroad, or from public or private research centers.
L'archive ouverte pluridisciplinaire HAL, est destinée au dépôt et à la diffusion de documents scientifiques de niveau recherche, publiés ou non, émanant des établissements d'enseignement et de recherche français ou étrangers, des laboratoires publics ou privés. 
CHERCHER, REPÉRER, AVANCER

Table ronde

\title{
L'AVENIR DES NÉGOCIATIONS COLLECTIVES
}

\author{
ARIMHE | « RIMHE : Revue Interdisciplinaire Management, Homme \& Entreprise »
}

2015/2 n 16 | pages 3 à 17

ISSN 2259-2490

Article disponible en ligne à l'adresse :

https://www.cairn.info/revue-rimhe-2015-2-page-3.htm

Distribution électronique Cairn.info pour ARIMHE.

(C) ARIMHE. Tous droits réservés pour tous pays.

La reproduction ou représentation de cet article, notamment par photocopie, n'est autorisée que dans les limites des conditions générales d'utilisation du site ou, le cas échéant, des conditions générales de la licence souscrite par votre établissement. Toute autre reproduction ou représentation, en tout ou partie, sous quelque forme et de quelque manière que ce soit, est interdite sauf accord préalable et écrit de l'éditeur, en dehors des cas prévus par la législation en vigueur en France. Il est précisé que son stockage dans une base de données est également interdit. 


\section{Table ronde}

\section{L’avenir des négociations collectives}

Une table ronde a été organisée par la Revue Interdisciplinaire Management, Homme(s) \& Entreprise, la RIMHE, le 17 juin 2014 à la Faculté de Droit de l'Université Paris V Descartes sur les négociations collectives. Elle était animée par Marc MORIN, Professeur en économie et sciences des organisations à l'ISC de Paris. Quatre intervenants ont bien voulu répondre à son invitation : Franck BIETRY, Maître de Conférences HDR en sciences de gestion à l'IAE de Caen, Jacques FREYSSINET, Professeur émérite en économie à l'Université de Paris 1 et Président du Conseil scientifique du Centre d'Études sur l'Emploi, Jacques ROJOT, Professeur émérite en sciences de gestion à l'Université de Paris 2, Fondateur et past-rédacteur en chef de la Revue de Gestion des Ressources Humaines et Président de la CIDEGEF, Christian THUDEROZ, Sociologue, Professeur des Universités à l'INSA de Lyon et chercheur au Centre Max Weber. Leurs échanges ont été enregistrés ${ }^{3}$ et des extraits ont été sélectionnés et retranscrits pour, une fois relus par leurs auteurs, être présentés dans cet article.

Marc MORIN : Je voudrais tout particulièrement remercier nos éminents invités. L'objet de ce débat est de s'appuyer sur leur expertise pour mieux comprendre ce qu'est le syndicalisme, la négociation collective et de son avenir. Les formes syndicales de demain, nous ne les connaissons pas forcément. Il y a des mutations organisationnelles ; les institutions bougent avec un petit nombre de militants, de syndicalistes. Tout cela, il faut le comprendre pour l'anticiper. On commence par Franck.

Franck BIETRY : Pour ma part, j’enseigne la Gestion des Ressources Humaines. Pour cette raison, je me suis quasi exclusivement concentré sur le syndicalisme au niveau de l'entreprise et plus particulièrement de l'entreprise privée. Cet intérêt vient d'un constat : on peut observer qu'en Amérique du Nord, il existe des chaires d'université entièrement dédiées à ces questions. Il existe des centres de recherche qui se consacrent uniquement à la problématique syndicale alors que l'influence des organisations syndicales en Amérique du Nord est peut-être moins importante qu'en France. Chez nous, les organisations syndicales restent très influentes malgré un taux de syndicalisation faible. Elles pèsent fortement sur notre économie, et a contrario on peut constater un désintérêt quasi total des gestionnaires à l'égard de ces questions de négociations et de relations collectives. Cela aboutit à ce que les étudiants qui se forment au métier de cadre n'entendent pas parler du syndicalisme durant leur formation. Ils vont donc le découvrir sur le terrain, généralement au moment des crises, en appliquant tous les stéréotypes de pensée pour traiter les problèmes. Il m'a donc semblé qu'il y a un paradoxe très fort et un manque. Dans ce cadre-là, je me suis plus particulièrement intéressé aux attitudes et aux comportements des salariés syndiqués dans l'entreprise beaucoup plus qu'à ce qui se passe au niveau des branches et des confédérations. Je me suis plus particulièrement

\footnotetext{
${ }^{3}$ La vidéo réalisée par le service multimédia de la DISI de l'Université Paris Descartes est en ligne sur le site de l'université : http://media2.parisdescartes.fr/ (rubriques : Droit, Ethique et entreprise, Tables rondes)
}

RIMHE - Revue Interdisciplinaire Management, Homme(s) \& Entreprise

$\mathrm{n}^{\circ} 16$ - mars/avril 2015 
Table ronde : L'avenir des négociations collectives animée par Marc MORIN avec Franck BIETRY, Jacques FREYSSINET, Jacques ROJOT et Christian THUDEROZ

penché sur le concept d'implication dans les deux sens nord-américains, c'est-à-dire à la fois le «joining» _ pourquoi certains salariés prennent une carte syndicale ? Et dans quelle organisation ?, mais aussi de «l'acting » : que font-ils de cette carte syndicale ?

Jacques ROJOT : Il est rassurant quelque part de constater que mon voisin a parlé d'un thème que je voulais moi-même évoquer. Il se trouve donc que nous nous rejoignons assez facilement. En ce qui me concerne, depuis le début de ma carrière universitaire je me suis intéressé à ce qu'on appelle les relations professionnelles en France, "industrial relations » aux États-Unis où j'ai fait une partie de mes études. Ensuite, le sujet n'étant plus à la mode, j'ai évolué vers les modèles théoriques de négociation étendus à divers champs en dehors du champ social, et puis vers la théorie des organisations, mais le point de départ était bien le modèle syndical, le fonctionnement du syndicat comme organisation. Le point que je voudrais souligner est que j'ai passé une bonne partie de ma carrière à essayer d'expliquer à des collègues étrangers pourquoi le modèle syndical français n'était pas une exception. Tout le monde, de Tokyo à San Francisco, me dit «mais chez vous, ce n'est pas pareil !». Ce n'est pas vrai. C'est pareil. Simplement, les mêmes problèmes se situent dans un cadre économique, social, idéologique, différent, donc trouvent des réponses relativement différentes au même type de problèmes. Qu'estce que c'est un syndicat ? Samuel Gompers, le père du syndicalisme américain, disait en 1890 : «c'est très simple : plus, plus, encore plus, maintenant ! »; cela est vrai partout. Le modèle syndical est fait pour améliorer la situation de ses constituants. L'on peut y ajouter une expression, une vocalisation, une fédération des mécontentements - il faut bien qu'ils aient une voix pour s'exprimer - et cette réalité existe partout. Mais, elle prend des traits particuliers, partout aussi ; il n'y a pas de modèle général pour lequel la France serait une exception ; il n'y a que des exceptions qui ont vécu, cru, plus ou moins prospéré en fonction du type de circonstances auxquelles ces organismes avaient à faire face. Alors c'est exact, et je reprendrai ce que mon voisin vient de dire. Il y a deux paradoxes à mon avis dans le syndicalisme français, mais qui s'expliquent pour des raisons historiques, économiques, idéologiques assez claires et simples. Le premier paradoxe, sur lequel je n'insisterai pas parce que cela vient d'être dit et très bien dit est qu'il est faible en effectifs et divisé, mais influent. Faible en effectifs, cela n'est pas récent. C'est actuellement beaucoup plus accentué que cela ne l'a été, mais jamais les effectifs du mouvement syndical français n'ont dépassé les 20-22 \% de la population active salariée même aux grands moments et à la meilleure époque. Comparés aux voisins européens ou transatlantiques, il a toujours été numériquement plus faible historiquement. On peut expliquer cela par une multitude de raisons dont la tradition de l'aristocratie de la classe ouvrière qui ne cherche pas à recruter, mais qui cherche à donner un exemple. Contrairement aux syndicalistes américains, les syndicats français cherchent plus des moyens d'action politiques qu'économiques. Faibles en effectifs depuis toujours et divisés depuis toujours. Pourquoi ? Parce que la structuration de la classe ouvrière en France s'est faite par vagues successives et non d'un seul coup, et en

RIMHE - Revue Interdisciplinaire Management, Homme(s) \& Entreprise

$\mathrm{n}^{\circ} 16$ - mars/avril 2015 
suivant un très long processus. La révolution industrielle est arrivée en France beaucoup plus tard, précédée d'une longue proto-industrialisation, et elle s'est déroulée beaucoup plus lentement sur une période beaucoup plus longue qu'en Allemagne, en Angleterre ou même en Belgique. Au lieu d'un mouvement ouvrier qui se construit très rapidement avec une industrialisation rapide et brutale, il y a eu des séries de composants successifs au fur et à mesure du temps qui viennent rejoindre une classe ouvrière en constitution quand les modes de production anciens et nouveaux coexistent, l'un remplaçant lentement l'autre. Au fil du temps, toute une série de catégories de gens différents sont venus s'agglomérer en une masse peu homogène, avec des intérêts et des idéologies forcément différents, voire divergents: vagues successives d'anciens employés agricoles, paysans ouvriers à temps partiel, travailleurs saisonniers, travailleurs migrants, femmes, compagnons, anciens artisans victimes des crises économiques du $\mathrm{XIX}^{\circ}$ siècle. Cette constitution de la classe ouvrière lente et par composantes, qui gardaient une identité différente, a forcément créé des attentes, des aspirations différentes, donc un mouvement syndical divisé. Un autre facteur de division sera les liens avec des mouvements sociaux et partis politiques d'inspiration opposée: socialistes révolutionnaires, anarcho-syndicalistes, socialistes réformistes, guesdistes-marxistes, etc. Ces liens sont en même temps un outil d'influence politique. Un mouvement donc faible en effectifs, divisé, mais influent. Cette influence persiste. Elle ne change pas. Il y a toute une série de moyens juridiques qui la maintiennent : les instances représentatives du personnel, le monopole de la signature des accords collectifs, le champ automatique d'application de l'accord dès qu'il est signé. Cela constitue d'ailleurs un taux de couverture par conventions collectives qui, malgré la faiblesse syndicale et le taux le plus bas de syndicalisation de l'OCDE, est le plus élevé de l'OCDE. De plus le mouvement syndical bénéficie d'une reconnaissance générale qui est largement incontestée, même si bien sûr les employeurs s'inscrivent en adversaires/partenaires. Le deuxième paradoxe est celui du dedans/dehors. Cela surprend beaucoup nos collègues étrangers, les syndicats français sont marqués par une rhétorique et des stratégies frappées au sceau du conflit, soit le conflit de classes pour les syndicats les plus radicaux, soit des conflits de positions difficilement réconciliables pour le clan réformiste. L'opposition est le premier réflexe. Si je reprends une rapide revue de presse venant de tous les azimuts syndicaux l'on relève régulièrement des termes tels que « cadeaux aux patrons », «défense des masses opprimées », « déclaration de guerre aux salariés ». On a une idéologie de changement de société du côté des syndicats, qu'ils soient radicaux ou réformistes. Leurs cadres d'ailleurs tendent à être relativement similaires à l'intérieur de chacune des organisations parce que l'on a tendance à s'y auto recruter entre gens partageant des idées identiques du fait du faible nombre des adhérents et des militants. D'autre part, il existe une longue tradition historique, qu'il faut bien rappeler, d'une classe ouvrière qui s'est constituée, qui a crû, qui a vécu en dehors de la 
Table ronde : L'avenir des négociations collectives animée par Marc MORIN avec Franck BIETRY, Jacques FREYSSINET, Jacques ROJOT et Christian THUDEROZ

société, comme rejetée par elle. L'ouvrage de Chevalier de 1958, Classes laborieuses, classes dangereuses ${ }^{4}$ montre bien comment le mouvement ouvrier s'est constitué dans une tradition de conflit en dehors de la société au lieu de s'agglomérer à des éléments de cette société, en répétition du modèle décrit par Tocqueville pour l'ancien régime entre noblesse et bourgeoisie. Donc les syndicats sont dehors, mais en même temps, ils sont dedans et bien dedans car les syndicats en France sont très institutionnalisés. Ils sont finalement les moteurs du système social. Ils sont présents à tous les niveaux des structures sociales. Ils administrent les systèmes de protection sociale. Ils font partie des comités économiques et sociaux, régionaux et d'Etat ; ils sont là présents partout. Voilà les deux paradoxes qui s'expliquent par les facteurs socio-économiques et historiques que j'ai voulu brièvement et incomplètement rappeler.

Marc MORIN : Il existe aujourd'hui d'importants travaux portant sur un climat social des négociations en France en l'occurrence particulièrement détérioré. En termes de théorie des jeux, cela veut dire qu'on tend à anticiper plus ou moins systématiquement l'autre comme ennemi, donc à adopter d'emblée vis-à-vis de lui des actions de provocation, qui vont souvent en retour conditionner des réactions également plus agressives de l'autre, et réciproquement. D'où des conflits souvent plus durs qu'ailleurs qui s'auto alimentent et gênent voire empêchent la découverte de réels compromis. Je pense aux travaux de Philippon, Algan et Cahuc. Les économistes conventionnalistes montrent également que ces phénomènes de représentations collectives et réciproques, liés à la culture, aux institutions léguées par l'histoire, interfèrent constamment dans les négociations sociales. Jacques ...

Jacques FREYSSINET : Je suis économiste. Mon intérêt pour la négociation collective, plus largement pour les systèmes de relations professionnelles est né en réaction contre le discours dominant chez les économistes orthodoxes selon lequel, dans le contexte de l'internationalisation, de la mondialisation, ensuite de l'écroulement des pays du socialisme réel, il y avait un modèle d'efficacité unique qui s'imposait à l'échelle mondiale, et que, de ce fait, un mouvement de convergence inéluctable était à l'œuvre, y compris dans les modes d'organisation du marché du travail. Ainsi existerait-il modèle idéal d'efficacité de la négociation collective dans une économie de marché mondialisée. Or, avec des nuances, l'observation en ce domaine prouve le contraire. Aucun mouvement majeur de convergence, me semble-t-il, ne peut s'observer entre les systèmes de relations professionnelles, au moins pour les pays industrialisés. Et donc l'interrogation est la suivante : comment se fait-il que dans des systèmes économiques qui fondamentalement tendent à avoir des caractéristiques très proches, les modes d'organisation sociale, en particulier les systèmes de relations professionnelles, continuent à reproduire _certes d'une façon évolutive_des spécificités très fortes. L'hypothèse que je vais caricaturer en quelques phrases au risque de faire frémir mes

\footnotetext{
${ }^{4}$ Chevalier L. (1978), Classes laborieuses, classes dangereuses, Paris, Le Livre de poche, coll. Pluriel, Plon RIMHE - Revue Interdisciplinaire Management, Homme(s) \& Entreprise $\mathrm{n}^{\circ} 16$ - mars/avril 2015 
voisins et l'auditoire, c'est que ces spécificités résultent de cocktails historiques différents qui se sont produits entre trois conceptions élémentaires de l'action syndicale, qui se traduisent par trois types de stratégie de la négociation collective. Il y a d'abord, on l'a évoqué, Samuel Gompers. C'est essentiellement un conflit de répartition dans une économie de marché dont on ne met pas en cause les règles du jeu. Simplement, on construit un rapport de forces qui permet d'exiger plus dans le conflit de répartition avec le patronat, un patronat dont on ne conteste pas le pouvoir de gestion. Le syndicat ne se mêle pas de la gestion, il lutte pour le partage : salaire, protection sociale, réduction de la durée du travail, etc. Deuxième composante, la composante "lutte de classes ». Il semble qu'elle existe encore. L'objectif du mouvement syndical c'est la transformation de la société, et les luttes sociales, les négociations qu'elles peuvent engendrer, ne sont que des étapes dans ce projet de transformation sociale. Le syndicalisme est dehors aussi longtemps qu'il ne sera pas dedans, c'est-à-dire aussi longtemps qu'il ne sera pas dans une société qui correspond à ses objectifs. Donc, la négociation collective traduit à un moment donné un rapport de forces ; c'est un armistice provisoire que, de part et d'autre, on est prêt à remettre en question si le rapport de forces se modifie. Et puis, il y a une troisième conception, qui est plus complexe. Après la Seconde Guerre mondiale, certains l'ont théorisé sous le terme ambigu de néo corporatisme. C'est l'idée selon laquelle dans les sociétés suffisamment développées, Etat, Patronat et Syndicats, dès lors qu'ils ont une vision d'ensemble de la société, sont capables de construire des compromis d'intérêt commun. On ne nie pas qu'au départ, leurs intérêts soient divergents, mais ils peuvent trouver des compromis à l'avantage de tous. Dans le vocabulaire courant, c'est l'idée d'accord «gagnant-gagnant». Il y a des origines historiques de ce mouvement. On le trouve beaucoup dans la doctrine sociale de l'Église, mais on le trouve aussi dans différentes formes de corporatisme qui ne sont pas forcément des corporatismes dictatoriaux. Il y a des variantes soft du corporatisme. Il est clair que selon les périodes de l'histoire, mais selon aussi les traditions culturelles, les systèmes politiques, ces trois composantes n'ont pas du tout pesé de la même façon dans chacun des pays, et il n'est pas très difficile d'en dessiner une carte, non pas avec des couleurs homogènes mais avec des dominantes. On voit bien comment le syndicalisme de lutte de classes a été longtemps la forme dominante dans les pays d'Europe du Sud, par exemple, avec toute une série de prolongements en Amérique latine. On voit bien que les pays anglo-saxons ont plutôt été les pays d'expérimentation du premier type de syndicalisme ; et on voit bien que les pays germaniques et nordiques relèvent plus du troisième, mais comme des illustrations de tendances : il ne faudrait surtout pas réduire une classification à des tiroirs dans lesquels on distribuerait les pays. Simplement il me semble que cette composante culturelle au sens fort, c'est-à-dire qui est située au centre des visions de la société, permet de comprendre comment aujourd'hui encore, face à des problèmes de nature économique qui peuvent apparaître comme en voie d'homogénéisation totale, les systèmes de relations professionnelles, et donc de négociations, restent à ce point hétérogènes dans leur niveau, dans leurs modalités, dans leurs résultats, et ceci même

RIMHE - Revue Interdisciplinaire Management, Homme(s) \& Entreprise $\mathrm{n}^{\circ} 16$ - mars/avril 2015 
Table ronde : L'avenir des négociations collectives animée par Marc MORIN avec Franck BIETRY, Jacques FREYSSINET, Jacques ROJOT et Christian THUDEROZ

dans des pays aussi proches que la Belgique et les Pays-Bas, ou l'Espagne et l'Italie. Des pays apparemment très voisins peuvent engendrer des systèmes de négociations collectives profondément différents, non pas de manière statique _c'est évolutif _, mais les spécificités sont durables.

Marc MORIN : C'est vrai que pour la France par exemple, certains observateurs étrangers et notamment Américains comprennent difficilement qu'un délégué syndical y négocie notamment, qu'ils soient ou non encartés et si oui quelles que soient leurs sensibilités, pour tous les salariés présents dans l'entreprise. Ce qui est vrai aussi du troisième niveau national et interprofessionnel des négociations bénéficiant à tous les salariés français. C'est très particulier. C'est bien là un exemple de forme institutionnelle particulière trouvant ses racines dans l'histoire, et dans laquelle s'encastrent des pratiques spécifiques de négociation sociale qui sont d'une certaine façon plus politisées qu'ailleurs. Christian...

Christian THUDEROZ : Je suis sociologue. Depuis trente ans, j'essaye de développer tant bien que mal_actuellement, j'essaye de les faire converger_, deux axes de réflexion. Le premier, que j'ai nommé «sociologie de la négociation», a produit un certain nombre d'ouvrages qui portent explicitement ce titre. Très peu de travaux de sociologues existent sur cette activité. Le fait qu'il y ait si peu de chercheurs dans les sciences sociales, et en sociologie plus particulièrement, à s'y intéresser dans le champ français est un paradoxe, puisque nous passons notre temps à nouer des compromis! Ce qui m'intéresse consiste d'abord à comprendre ce peu de propension, ici dans notre pays _si je reprends l'expression de notre regretté Jean-Paul Jacquier, décédé en mai dernier : «La France : un pays qui n'aime pas négocier »_, à ne pas cultiver le compromis ; d'où provient ce non-amour de la négociation? Ma seconde réflexion sur la négociation consiste à comprendre l'originalité de ce mécanisme décisionnel. Un certain nombre d'individus ont des intérêts différents, des points de vue différents, des objectifs à atteindre différents, et, néanmoins, ils arrivent à construire, tant bien que mal la plupart du temps, des accords. Ceux-ci permettent la poursuite des actions de chacun et du cours des actions collectives. Or, ces individus ont à leur disposition _c'est particulièrement net dans le cas français_, d'autres mécanismes décisionnels possibles : le recours au juge, la décision unilatérale, le vote, etc. Pourquoi ces individus choisissent-ils plutôt ce mécanisme décisionnel qu'est la négociation ? Voilà une question sociologique qui me semble intéressante. Cela occupe $50 \%$ de ma vie de chercheur. L'autre $50 \%$ est constitué de ce que j'ai nommé « sociologie de l'entreprise ». Nous étions quelques-uns, avec Denis Segrestin au début des années 90, à oser affirmer que l'entreprise est une institution cardinale, fondamentale de notre société moderne, et il nous importait que les sociologues réfléchissent à cette question et à ses conséquences, théoriques et pratiques. J'essaye actuellement de faire converger ces deux axes de réflexion, négociation et entreprise. Ce que je tente, c'est un peu de renouer avec les débats que nous avons tous un peu délaissés, autour de ce que l'on appelait alors « la démocratie industrielle ». Une

RIMHE - Revue Interdisciplinaire Management, Homme(s) \& Entreprise

$\mathrm{n}^{\circ} 16$ - mars/avril 2015 
Table ronde: L'avenir des négociations collectives animée par Marc MORIN avec Franck BIETRY, Jacques FREYSSINET, Jacques ROJOT et Christian THUDEROZ

démocratie dans l'entreprise, aujourd'hui qu'est-ce que ça signifie ? Isabelle Ferreras dans son ouvrage ${ }^{5}$, Gouverner le capitalisme?, propose d'instaurer le bicaméralisme, c'est-à-dire de créer deux chambres dans l'entreprise : une première chambre, celle des apporteurs en capitaux, et une seconde chambre, celle des investisseurs en travail, sur le mode de la démocratie usuelle comme, par exemple dans le cas français, une Assemblée et un Sénat. Voilà une manière à mon avis tout à fait heureuse _il y a eu d'autres initiatives ces dernières années ; je pense aux travaux de Hatchuel et Segrestin ${ }^{6}$ et leur proposition d'un contrat d'entreprise_, de s'interroger sur les mécanismes de décisions dans l'entreprise à la lumière de ce qu'on peut imaginer du point de vue de la démocratie.

Marc MORIN : Je te remercie. La question que l'on pourrait poser alors est celle des rationalités. Franck, les rationalités de celui qui prend une carte, qui devient délégué, de ceux qui l'accompagnent, qui vont militer..., à quoi ça sert le syndicalisme ? Les économistes de la tradition orthodoxe nous disent que plus ou moins systématiquement les syndicats «ça mange de la rente », autrement dit finalement qu'ils diminuent par leurs interventions le taux de croissance, l'emploi, qu'ils freinent la bonne évolution du système. Il y a toutefois au moins deux discours qui sont radicalement différents. Les économistes keynésiens ou postkeynésiens, avec de grands anciens qui vont dire que le syndicalisme « produit de la rente»: effets d'entrainement de la demande de consommation, de l'investissement, etc. Et qu'au niveau de l'Union Européenne, ils pourraient participer à la relance s'il existait en la matière une réelle coordination politique. Et puis, il y a aussi une autre École qui est intéressante. C'est celle de Freeman et Medoff qui, dans la foulée d'Hirschman, nous dit que le syndicalisme et la négociation doivent être regardés comme participant d'un fait social total. Que dans nombre de cas, les effets par exemple inflationnistes des revendications sont largement plus que compensés par de nombreuses chaines d'effets positifs à l'intérieur de l'entreprise, et plus généralement à l'intérieur de la société. Et qu'à l'inverse l'absence de négociation génère un certain nombre d'effets contre-productifs. Des travaux montrent par exemple que, quand il y a un délégué syndical présent et actif dans une entreprise, les salariés sont plus confiants dans l'avenir, qu'il y a une relation entre la confiance et la productivité, que les performances peuvent être plus fortes là où il $\mathrm{y}$ a une culture organisationnelle forte, et notamment une cohésion sociale en bonne partie produite par la possibilité de prendre la parole au sens d'Hirschman. Alors Franck, les rationalités du syndicalisme ?

Franck BIETRY : A quoi sert un syndicat en 2014 ? La question est vaste. Pour essayer d'y répondre, je vais la renverser. Au niveau de l'entreprise, que se passe-t-il quand il n'y a pas de représentation collective instituée ou qu'est ce qui risque de se passer ? Il y a certainement un confort de gestion plus grand pour le dirigeant. Il n'est pas contesté

\footnotetext{
${ }^{5}$ Ferreras I. (2012), Gouverner le capitalisme ?, Paris, PUF.

${ }^{6}$ Segrestin B., Hatchuel A. (2012), Refonder l'entreprise, Paris, Coédition Seuil/La République des Idées. RIMHE - Revue Interdisciplinaire Management, Homme(s) \& Entreprise $\mathrm{n}^{\circ} 16$ - mars/avril 2015 
Table ronde : L'avenir des négociations collectives animée par Marc MORIN avec Franck BIETRY, Jacques FREYSSINET, Jacques ROJOT et Christian THUDEROZ

dans ses décisions, en tous cas, beaucoup moins et pas de manière officielle. Donc, cette situation est certainement plus confortable psychologiquement pour lui. Mais il y a plusieurs revers à la médaille si je puis dire. La première antithèse que l'on peut développer à ce sujet est que la désertification syndicale ne supprime pas les problèmes au travail. La plupart des problèmes au travail ne naissent pas avec l'apparition des organisations syndicales. Ce serait une erreur fondamentale de considérer une entreprise comme une communauté harmonieuse, une bande de bons copains, une famille au sein de laquelle tout le monde s'entend. Dans le monde du travail, il existe des tensions qu'on le veuille ou non. Ces tensions sont consubstantielles à la situation professionnelle. L'une d'entre elles est particulièrement forte. Les Américains en ont largement débattu. Elle oppose, d'un côté, un besoin d'efficience de l'entreprise c'est-à-dire de profit. $\mathrm{Ce}$ besoin est tout à fait légitime quand on accepte l'idée qu'on est en économie de marché. D'un autre côté, il y existe aussi un besoin de sécurité et de justice sociale qui est exprimé par les salariés. Lui aussi est tout à fait légitime dès l'instant où on accepte l'idée que le salarié est un facteur de production, mais aussi et d'abord un être humain. Ces tensions, elles existent donc. Si elles ne sont pas canalisées par une représentation collective, elles risquent fort de se transformer en conflits, en conflits larvés. Leurs modes d'expression sont alors beaucoup plus pernicieux et beaucoup plus pénalisants pour l'entreprise. Quand ces tensions débouchent sur un conflit ouvert, non plus larvé, et que ce conflit n'est pas encadré par une représentation collective, le directeur des ressources humaines devra trouver avec qui négocier. Qui est l'interlocuteur légitime dans ce cas ? Quel est son statut ? Cette légitimité reste à démontrer. A défaut, les accords de fin de conflit qui peuvent être trouvés sont, pour le coup, beaucoup plus instables parce que leur contenu peut être contesté par les autres salariés. La fonction d'un délégué syndical dans une entreprise, ça sert aussi à incarner ce que Durkheim appelait un "appareil de socialisation ». Il s'agit pour lui de faire prévaloir une morale, une discipline collective, qui va aussi permettre la reprise du travail et éviter la prolifération de revendications irréalistes. Le troisième argument qui justifie la présence syndicale est purement juridique. Un autre problème se pose en effet quand il n'y a pas de représentants syndicaux dans l'entreprise : comment adapter le Code du travail aux particularités locales? Plus généralement, comment saisir la multitude d'opportunités qui sont offertes dans le Code du travail aux directeurs des ressources humaines, mais qui sont conditionnées pour un grand nombre d'entre elles à la signature d'un accord collectif ? Pour signer un accord, il faut être deux qu'on le veuille ou non. Je veux dire par là qu'il faut bien un interlocuteur. En quatrième lieu, il faut comprendre qu'en l'absence d'organisation des salariés instituée, une croissance quasi exponentielle des coûts de transaction risque d'être observée. Il va falloir au DRH négocier individuellement, salarié par salarié. On remplace en fait une seule négociation collective par une multitude d'entretiens individuels. Et en cas d'insuccès pour le salarié du fait de son faible pouvoir, la tentation est grande pour lui de se replier sur lui-même et d'instrumentaliser l'entreprise à des profits strictement personnels. Derrière la présence

RIMHE - Revue Interdisciplinaire Management, Homme(s) \& Entreprise

$\mathrm{n}^{\circ} 16$ - mars/avril 2015 
Table ronde: L'avenir des négociations collectives animée par Marc MORIN avec Franck BIETRY, Jacques FREYSSINET, Jacques ROJOT et Christian THUDEROZ

syndicale réside donc une vraie source de légitimité qui intéresse autant le salarié que le fonctionnement de l'entreprise. Vouloir l'analyser uniquement en termes de captation de rentes, comme certains économistes classiques le font, c'est trahir une réalité beaucoup plus complexe qu'il n'y paraît.

Marc MORIN : Les économistes institutionnalistes et notamment conventionnalistes tendent effectivement à donner toute leur importance à des avantages pour l'entreprise nés des négociations dont certains transitent dans la tradition de Freeman et Medoff par des chaînes de réaction hors prix. Les effets d'augmentation de la productivité via le supplément de motivation qu'entraînent la diminution de l'incertitude sur les salaires et la reconduction des contrats de travail, liés à la présence rassurante des syndicats dans les entreprises, sont de cet ordre. Les effets plus généralement liés à la construction, via la culture des négociations, de davantage de confiance sociale le sont également. A la condition naturellement que cette culture se développe. C'est là, Jacques, un de tes thèmes d'intérêt privilégié...

Jacques ROJOT : Je suis tout à fait d'accord avec ce qui a été dit, avec la seule opposition qu'il n'y a, à mon sens, aucune raison qu'une entreprise soit gouvernée de façon démocratique. Une entreprise n'est pas un parti politique ; ce n'est pas un pays. Il n'y a aucune raison impérative qui l'impose. Tout ce qu'on demande à l'entreprise, c'est de fonctionner. Ceci dit, il est parfaitement exact que la négociation collective a l'avantage énorme de remplacer $\mathrm{X}$ négociations individuelles, autant qu'il y a de salariés. C'est un avantage considérable. Dans ce sens, le syndicalisme est extrêmement utile. Maintenant, il y a une autre question qui avait été posée et qui est intéressante à considérer en France : que gagne un salarié à se syndiquer en France ? Rien du tout ! Sinon le droit de payer une cotisation. De toute façon, il est couvert par le mode d'extension des conventions collectives et par le fait que, du fait de la Loi, une convention une fois signée par l'employeur, s'applique à tout le monde. Il bénéficie automatiquement de tous les biens et services qui ont été négociés par le syndicat pour ses membres. Il n'a aucune raison de se syndiquer sauf des raisons qui sont de nature idéologique ou morale. Ceci dit, bien évidemment, en pratique, le syndicat est un poil à gratter permanent pour l'employeur, et il lui crée des coûts supplémentaires, mais lui économise l'atomisation des revendications, les problèmes de sortie de crise quand il n'y a personne avec qui négocier. Par ailleurs, il est soumis à toutes les obligations du Code du travail qui imposent avec plus ou moins de succès la présence des organisations syndicales pour obtenir certains résultats. D'autre part, le cadre institutionnel français est totalement défavorable à la négociation collective pour toute une série de raisons. Un indicateur simple le démontre : si vous prenez deux ouvrages de référence du droit du travail : un français, celui de Jean-Emmanuel Ray ${ }^{7}$; un américain, celui de William B.

\footnotetext{
${ }^{7}$ Ray J.-E. (2010), Droit du Travail, Droit vivant, 2010/2011, Paris, Editions Liaisons Sociales RIMHE - Revue Interdisciplinaire Management, Homme $(s)$ \& Entreprise $\mathrm{n}^{\circ} 16$ - mars/avril 2015 
Table ronde : L'avenir des négociations collectives animée par Marc MORIN avec Franck BIETRY, Jacques FREYSSINET, Jacques ROJOT et Christian THUDEROZ

Gould $^{8}$, dans le manuel français, sur 800 pages on en compte 90 portant sur la négociation collective ; dans le manuel américain, sur 440 pages il y en a 150 portant sur ce sujet; dans un cas : $10 \%$, dans l'autre : $33 \%$. Cela veut dire que, dans le système français, la négociation collective est quelque chose de tout à fait secondaire. Par contre toute la partie législative, elle, est considérablement importante. Toute une série de choses qui chez nos voisins seraient réglées par des négociations collectives, sont en France fixées, établies par la Loi. Il y a très peu de place pour la négociation collective dans le système institutionnel français, nonobstant la volonté régulièrement affichée des pouvoirs publics de la promouvoir, pour d'ailleurs immédiatement légiférer encore plus abondamment. Certes nombre de textes conventionnels sont signés, mais, d'une part ils sont souvent au niveau de la profession ou de l'interprofession, et d'autre part, souvent aussi, à de notables exceptions près, mais qui restent des exceptions, ils reproduisent la Loi. Une dialectique Loi/accords nationaux interprofessionnels s'est d'ailleurs récemment développée. Il faut noter que le mauvais fonctionnement de la négociation collective et de ses résultats ne pose pas beaucoup de problèmes en période de prospérité, quand les entreprises peuvent payer et veulent payer (des salaires) : il n'y a alors pas de problème. Les entreprises veulent attirer et conserver une main-d'œuvre de qualité. Donc on signe une série d'armistices successifs. On reste peut-être en assez mauvais termes, mais on signe quoiqu'on pense de l'autre partie. En période de crise, les choses sont beaucoup plus difficiles parce qu'un mécanisme qui fonctionne plus ou moins chaotiquement, mais qui fonctionne pour le partage d'un surplus, va se bloquer dès qu'il s'agira de négocier des concessions réciproques ou de négocier quelque chose de plus compliqué. Donc cet environnement institutionnel négatif pour la négociation collective pose, dans l'environnement économique dans lequel nous sommes actuellement, des problèmes encore plus considérables.

Jacques FREYSSINET : Je suis tout à fait convaincu par les arguments qui ont été avancés pour expliquer pourquoi au fond une direction avait intérêt à avoir des syndicats, ou plus largement des représentants légitimes du collectif en face d'elle. Si on regarde l'histoire, force est de constater que les directions d'entreprise se sont battues aussi longtemps qu'elles l'ont pu pour ne pas avoir de syndicats en face d'elles, pour ne pas avoir à négocier, je dis bien au niveau de l'entreprise. La négociation n'est apparue historiquement que comme négociation de fin de conflit, lorsqu'un syndicat avait réussi à s'imposer dans l'entreprise contre la volonté de l'employeur. Je pense qu'il faut partir de là : il n'y a pas eu historiquement des employeurs rationnels qui ont découvert les avantages qu'ils avaient à avoir un syndicat pour négocier avec lui. Ceci dit, les choses ont changé, et ce qui me parait aussi important comme thème de réflexion, c'est le fait qu'à partir des années 90 , avec des prolégomènes dans les années 80 , on voit au contraire dans un certain nombre de cas les employeurs être à l'initiative de la

\footnotetext{
${ }^{8}$ Gould W.J. IV (2013), A Primer on American Labor Law, Cambridge, Cambridge University Press RIMHE - Revue Interdisciplinaire Management, Homme(s) \& Entreprise $\mathrm{n}^{\circ} 16$ - mars/avril 2015 
négociation. Il me semble qu'il n'y a pas de précédents historiques. Et ce mouvement est à l'œuvre aujourd'hui, où l'on voit que les directions d'entreprise, sans que ce soit systématique _ il faudrait discuter des variables explicatives _, choisissent de prendre l'initiative de la négociation pour, à mes yeux, obtenir une légitimation par les syndicats de sacrifices qui sont principalement des sacrifices salariaux, qui peuvent aussi être des sacrifices en termes de durée du travail, en termes de prestations sociales complémentaires, parce qu'elles considèrent que le rapport des forces leur permet d'obtenir l'acquiescement syndical à des concessions de ce type. Notamment par une stratégie de mise en concurrence, soit au sein d'un groupe industriel, des unités de production du pays ou à l'échelle internationale, soit par des menaces de délocalisation, par des menaces d'externalisation, et cette stratégie dans le contexte des 20 dernières années semble avoir remarquablement réussi. Je pense qu'il faut introduire une nouvelle dimension dans les stratégies de direction d'entreprise à l'égard de la négociation collective. Je pense qu'elles sont devenues une arme offensive qui permet à partir de, on pourrait dire «d'un chantage à l'emploi », ou sans utiliser des termes péjoratifs «d'objectifs d'emploi », d'obtenir en contrepartie des sacrifices en termes de coût du travail. Je crois que c'est un dilemme que les organisations syndicales ont la plus grande difficulté à gérer. Elles y sont affrontées dans les différents pays. Elles tiennent des discours très différents au niveau des confédérations. Les pratiques à la base permettent beaucoup plus difficilement de distinguer les différentes organisations syndicales. Ce sont beaucoup plus les contextes locaux que les appartenances syndicales qui commandent les choix des délégués syndicaux. Mais cela me semble modifier qualitativement de façon assez profonde l'enjeu de la négociation. Nous ne sommes plus dans une négociation de conquête de droits nouveaux. Nous sommes dans une négociation de concessions, où des engagements plus ou moins durables, plus ou moins contrôlables pour l'emploi sont échangés contre des sacrifices dans les autres aspects de la relation d'emploi : en gros, le salaire, la protection sociale et la durée du travail.

Marc MORIN : C'est quelque chose qui s'observe tout particulièrement au niveau de l'Union européenne, avec les phénomènes de délocalisation compétitive. Aujourd'hui, à l'échelle des 28 pays, les négociations sont davantage des négociations défensives que des négociations de compromis en termes de concessions réciproques. Cela soulève la question du développement de la Confédération européenne des syndicats et du développement d'un dialogue social à cette échelle.

Christian THUDEROZ: Puisque nous sommes là pour débattre, je me permettrai d'avoir une approche peut-être un peu décalée par rapport à celle de Jacques. Je partirai de ce que disait Allan Flanders, un sociologue anglais, au milieu des années 60. Il essayait de comprendre ce qu'est une négociation collective dans l'entreprise et il notait que, contrairement à ce qu'on croit, le syndicat n'est pas d'abord un agent économique, mais plutôt un acteur politique. Il employait cette expression, je crois, pour désigner ces personnes qui négocient au sein de l'entreprise : ce sont des législateurs, disait-il. Je

RIMHE - Revue Interdisciplinaire Management, Homme(s) \& Entreprise $\mathrm{n}^{\circ} 16$ - mars/avril 2015 
Table ronde : L'avenir des négociations collectives animée par Marc MORIN avec Franck BIETRY, Jacques FREYSSINET, Jacques ROJOT et Christian THUDEROZ

partirai de cette idée : la négociation collective peut être comprise comme une activité où un certain nombre d'individus définissent ensemble des règles. C'est une première réponse à la question : pourquoi négocier dans l'entreprise ? C'est parce qu'il faut bien définir des règles, résoudre des problèmes, terminer des conflits. La question devient dans ce cas-là : pourquoi beaucoup de négociations, comme vient de le rappeler Jacques, sont des négociations de fin de conflit ? Parce que 99,9\%, me semble-t-il, des décisions à prendre dans l'entreprise le sont au sein d'un processus de concertation, un processus de consultation, un processus d'ajustement, et qu'il y a très peu d'occasions où les décisions à prendre conduisent à des conflits. C'est un phénomène intéressant à étudier : d'où provient ce blocage ? Et pourquoi à un moment donné les conflits surgissent ? Si on a une lecture, qui est la mienne, consistant à considérer la négociation comme un processus de décision, il me semble intéressant d'examiner les productions de ces processus de négociation. Qu'est-ce que cette activité produit? Quels effets cela génère ? Cela produit beaucoup de choses ! Il y a des effets d'apprentissage, ce que j'appelle « une dynamique générative ». Par l'écoute des arguments de l'autre et par la production de ses propres arguments, et la nécessité d'ajuster ses arguments avec ce qui vient d'être énoncé par l'adversaire, cela fait que les individus reconsidèrent le problème, leur position, leurs objectifs ; ils s'adaptent, ils cheminent différemment, etc. J'ai regardé le nombre d'accords d'entreprise signés en France en 2012: 48 000, et le nombre d'entreprises signataires d'un accord : 31000 . Quand on fait le rapport, on s'aperçoit qu'en moyenne, c'est un accord et demi signé dans les entreprises signataires. Cela veut dire qu'il y a un certain nombre d'entreprises qui ont signé un seul accord ; mais cela veut aussi dire qu'un certain nombre d'autres entreprises ont signé plus d'un seul accord, parfois jusqu'à 3 accords ou des avenants à des accords existants. Ce serait intéressant d'examiner les caractéristiques des entreprises qui ont signé plus d'un accord. Je fais l'hypothèse que dans de telles entreprises une "dynamique générative » est à l'œuvre. A partir du moment où dans ce type d'entreprise on a résolu un certain nombre de problèmes, on a pris des décisions ensemble dont une partie concerne des sujets importants, alors six mois plus tard, un an plus tard, deux ans plus tard, un certain habitus s'est créé. Bien sûr, tout cela est chaotique, puisque souvent l'acteur syndical est stable, alors que les acteurs gestionnaires, du fait de leurs carrières, quittent l'entreprise et qu'il y a donc, peut-être, des effets de mémoire et de transmission. Du coup, que penser de cette distinction : négociations de concessions versus négociations de droits nouveaux? La première chose est : toute négociation par définition est de concession! Une négociation, c'est un abandon réciproque d'un certain nombre de prétentions. C'est, je pense, une définition acceptable d'une négociation. Le compromis, qui est une des productions majeures d'un processus de négociation, c'est bien le fait que les deux parties concèdent pour arriver néanmoins à satisfaire une partie de leurs objectifs initiaux. Mais, dans le même temps, il peut arriver qu'il y ait obtention de droits nouveaux dans des négociations concessives. «Droits » pris ici au sens large. Mais je pense que dans un certain nombre d'entreprises, les syndicalistes sont confrontés à un

RIMHE - Revue Interdisciplinaire Management, Homme(s) \& Entreprise

$\mathrm{n}^{\circ} 16$ - mars/avril 2015

-14 - 
problème d'arbitrage et au dilemme classique: l'acceptation d'une perte, pour l'obtention d'un gain. Evidemment, tout cela est éminemment subjectif. Si jamais ces syndicalistes signent de tels accords, c'est que leurs priorités seront satisfaites, mais au détriment de divers autres choses, qu'ils peuvent donner à l'employeur. Conserver un certain nombre de sites de production en France vaut bien, me semble-t-il, je pense ici aux signataires des accords de Peugeot et de Renault l'an dernier, l'abandon d'une heure de travail par semaine ou un certain nombre de choses jugées, dans l'immédiateté de la négociation de l'accord, moins importantes que ce qui est obtenu. C'est pour cela que toute négociation étant concessive, il faut peut-être réexaminer la question de : qu'est-ce qui est obtenu par les deux parties? De ce point de vue, nous sommes, pour l'ensemble des acteurs sociaux _ patronat, syndicats et Etat_en France, très mal outillés. Cet art des concessions est quelque chose qui n'est guère élucidée dans notre pays.

Jacques FREYSSINET : Pour éviter de partir sur de faux débats. Il y a un débat surement, mais d'abord je suis complètement d'accord pour dire qu'une négociation se termine sur des concessions réciproques. J'ai employé le terme de négociation de concessions parce que c'est le terme qu'ont introduit nos collègues anglo-saxons dans les années 80 pour désigner un type particulier d'évolution, mais tu as raison, toute négociation se termine par des concessions. Ce qui à mes yeux a beaucoup changé, c'est la nature des concessions réciproques. Ce que concède l'entreprise aujourd'hui, ce sont des engagements sur l'emploi plus ou moins durables, plus ou moins fermes. Ce que concèdent les syndicats, ce sont des sacrifices salariaux ou para-salariaux. De toute façon les accords se signent. Ce qui montre qu'il y a des organisations syndicales qui estiment que ce compromis vaut mieux que pas de compromis. Il ne faut pas se tromper de débat et bien le localiser.

Christian THUDEROZ : Effectivement, j'ai été incomplet dans mon raisonnement. C'est pour cela que je fais bien la distinction entre deux types de concessions. Il y a d'abord le renoncement à une prétention : je voulais 10 , je me contente de 5 . Et puis il y a un second type de concession _ et effectivement on a tendance à confondre les deux_ c'est l'abandon d'un droit déjà acquis, ou d'un bien que l'on possède. La plupart du temps, les processus de négociation alternent un peu ces deux dimensions-là : renoncement à une prétention, et abandon. Que les autres acteurs fassent seulement des « engagements à », effectivement, on peut affirmer qu'il y a là une certaine asymétrie. Mais là je parie sur le fait que, quand un acteur signe un accord dans lequel il stipule qu'il s'engage à agir_c'est la définition même du mot compromis : le fait de promettre, et de s'engager à tenir cette promesse_ quand il signe un tel accord, il ne peut se soustraire ensuite à cet engagement. Qu'est-ce qu'un compromis ? C'est le fait de dire : je promets, je m'engage à faire, et on le fait publiquement dans des accords que l'on signe et qui sont rendus publics. C'est extrêmement difficile pour cet acteur-là de se désengager et ne plus agir conformément à sa promesse. C'est là l'une des originalités de ce mécanisme décisionnel qu'est la négociation : en tant que dispositif collectif, il

RIMHE - Revue Interdisciplinaire Management, Homme(s) \& Entreprise $\mathrm{n}^{\circ} 16$ - mars/avril 2015 
Table ronde : L'avenir des négociations collectives animée par Marc MORIN avec Franck BIETRY, Jacques FREYSSINET, Jacques ROJOT et Christian THUDEROZ

comprend un engagement mutuel devant l'autre à entreprendre l'action promise.

Marc MORIN : Une question dans la foulée sur la désyndicalisation : est-ce que qu'on peut considérer que c'est là un phénomène irréversible? Quel est le rôle des gestionnaires ? Au sein des entreprises, il y a toute une structure de pouvoir, et certains salariés n'osent pas s'engager par peur des mesures de rétorsion. Il y a même un autre phénomène, c'est le processus d'individualisation, de « dé -collectivisation » comme le souligne Robert Castel : qu'est-ce qui se passe dans le tissu social aujourd'hui et au niveau de la gestion de l'entreprise?

Franck BIETRY : Je voudrais nuancer cette idée de crise du syndicalisme qui traîne dans bon nombre d'ouvrages. Je vais faire pour cela une thèse/antithèse une nouvelle fois. Effectivement, on observe une crise du syndicalisme si on considère le taux de syndicalisation. C'est vrai qu'afficher $5 \%$ de syndiqués dans le privé constitue une marque de crise, a fortiori quand on constate dans le même temps qu'on est au dernier rang des pays de l'OCDE. On peut dire aussi que le syndicalisme est en crise au regard du taux d'abstention aux élections professionnelles qui oscille entre 60 et $70 \%$. Enfin, la crise s'observe également dans l'impuissance relative des organisations syndicales à lutter contre ce qu'on appelle « les plans de sauvegarde de l'emploi », autrement dit les réductions d'effectifs, et leur puissance finalement très relative à "capter une rente », c'est-à-dire à obtenir des augmentations de salaire à la hauteur de ce qu'attendent les salariés. Donc, oui, de ce point de vue, on peut dire qu'il y a une crise du syndicalisme. Maintenant, je souhaiterais quand même relativiser fortement cette idée pour plusieurs raisons: d'abord, le Professeur Rojot nous l'a rappelé, parce que le syndicalisme français n'a jamais été un syndicalisme de masse, mis à part dans des périodes très précises comme au moment du Front populaire. Ensuite, parce que la première étude que j'ai trouvée au sujet de la crise du syndicalisme date du début du XXe siècle. Ce ne serait donc pas un phénomène conjoncturel. Enfin parce que l'influence de ces organisations reste quand même considérable. Christian Thuderoz nous l'a dit : « 48000 accords signés dans les entreprises en $2012 »$; dans les branches, 1236 textes signés au cours de la même période. Cela signifie une moyenne de 3 textes signés par branche. Au total, le Professeur Rojot a raison de rappeler que «97\% des salariés sont couverts par une convention collective ». Je ne vais pas égrener non plus la liste de tous les accords interprofessionnels importants qui ont été signés en France, mais ils sont nombreux chaque année. Ils sont pour la plupart d'entre eux repris quasi in extenso dans les textes de loi donc par le gouvernement. Cela s'explique notamment par le renforcement du nombre des thématiques de négociation légalement obligatoires : la GPEC (obligation triennale maintenant), le handicap, l'égalité professionnelle, la prévoyance, l'épargne salariale. Donc, bien sûr on peut regarder du côté du taux de syndicalisation et dénoncer une crise. Mais à mon avis, il est beaucoup plus pertinent de l'analyser en termes de crise du militantisme. En France, on a en effet affaire à un syndicalisme par procuration avec des salariés non encartés, mais intéressés et souvent timorés. Il s'agit beaucoup plus

RIMHE - Revue Interdisciplinaire Management, Homme(s) \& Entreprise

$\mathrm{n}^{\circ} 16$ - mars/avril 2015 
d'une crise de l'engagement dans le syndicat que du syndicat en tant que formation institutionnelle. Les relations professionnelles, le système syndical, lui, il produit: il produit des règles procédurales. Il produit aussi des règles distributives. Donc si on change d'angle de vue, on doit sacrément relativiser ce phénomène dit de «crise du syndicalisme ». La véritable crise du syndicalisme concerne à mon avis d'autres pays que le nôtre. Les États-Unis, par exemple, sont confrontés actuellement à un taux historiquement bas de syndicalisation - 6,5\% - et à une couverture conventionnelle très étroite. Méfions-nous toutefois de ces comparaisons internationales. Les indicateurs sont souvent biaisés car les formes syndicales sont très différentes. Seuls les pays qui ont opté pour le modèle de Gantt s'en sortent : ceux qui ont opté pour un syndicalisme de services qui conditionne l'accès à certaines prestations de services telles que la sécurité sociale, l'indemnisation chômage, etc., et conduit à la détention d'une carte syndicale. Est-ce qu'on peut se comparer ces situations-là ? Je n'en suis pas convaincu.

Jacques ROJOT : Je voudrais apporter une autre remarque et puis mentionner une distinction. Ma remarque est relative à votre exposé de tout à l'heure considérant qu'il n'y a aucun employeur qui rationnellement n'a créé un syndicat chez lui : Biensûr! Mais je crois qu'il faut distinguer le syndicalisme dans l'entreprise et hors entreprise. Il est clair qu'un employeur, et surtout un petit employeur, a presque toujours préféré, traditionnellement, et préfèrera peut-être le plus souvent ne pas avoir de syndicats chez lui, parce que, surtout en France, c'est une remise en cause de son autorité, c'est une négation de ses pouvoirs. Suivant l'adage traditionnel des employeurs français : «le syndicat a sa place, mais à sa place », et «à sa place », c'est dehors. Autant il est légitime dehors, autant on préfère ne pas en avoir chez soi. Sauf que : quand la Loi a donné - et c'est l'importance considérable de la Loi en France par rapport à la négociation collective - toute une série de droits aux salariés en tant qu'individus, alors il devient plus commode d'avoir un syndicat que de négocier individuellement. La distinction est celle particulièrement caractéristique en France avec notre absence d'entreprises de taille moyenne : celle entre grandes entreprises/petites entreprises. Il n'y a pas de doute que dans les petites entreprises, le droit du travail purement et simplement n'est souvent pas bien appliqué. Pourquoi ? Pas par méchanceté, pas par brutalité, mais par ignorance complète, totale, des deux côtés. Le Code du travail est trop compliqué. Si on n'est pas un expert du droit du travail tant du côté des représentants syndicaux que du côté de l'employeur, il est impossible de l'appliquer parce qu'on ne le connait pas bien et qu'on n'a pas le temps de s'en occuper. Donc la situation dans les petites entreprises est certainement extrêmement différente de la situation dans les grandes entreprises, où le syndicat se trouve, comme cela vient d'être dit très justement, tout à fait institutionnalisé avec un rôle extrêmement important de - non pas de cogestion parce qu'on n'est pas en Allemagne - mais de mise en place d'un certain nombre de décisions.

Marc MORIN : Il me reste à vous remercier. 